\title{
The Harm of Harmonic in Power Distribution Network and Its Equipment
}

\author{
Pang Yanjun, Liu Peng, Wang Xue, Tong Rui, Zhao Xinhua, Yang Yingying, \\ Jiao Hongzhi, Cao Huiyan, Xu Mei, Wang Lei, Zhu Wenlong \\ Fushun Power Supply Company, Liaoning Electric Power Company Limited, State Grid, China,
}

fushunpowersupply@163.com

Keywords: harmonic; electric energy loss; distribution network; equipment

\begin{abstract}
Through several examples, describes the harmonic distribution network and its equipment hazards. Harmonics can cause distribution transformers demonstration power loss and additional loss increases, resulting in reduced aging transformer oil and insulation properties, triggering a distribution transformer discharge breakdown or burn accidents; harmonics is amplified in a capacitor bank, and cause the capacitor bank fuse group burst, tripping and other accidents; harmonics cause the motor to reverse, vibration, noise and temperature; harmonic electromagnetic voltage transformer prone to occur ferromagnetic resonance over-voltage; harmonics resulting in increased distribution network line losses. Awareness and understanding harmonics, harmonic provide for effective governance scientific theory.
\end{abstract}

\section{Introduction}

Nonlinear load and impact load when produce can produce harmonics, and the distribution of injection, if is the injection of harmonic frequency $(50 \mathrm{~Hz}) 2,3,5,7$ times the size of an integer, is the high frequency[1-3]. If the injection of harmonic frequency $(50 \mathrm{~Hz}) 1 / 2,1 / 3$, several times, $2 / 3$, $2 / 5,1 / 5$ is the frequency division; Harmonic pollution to the power system is not allow to ignore, because electric furnace, electric arc furnace, electrolytic furnace, intermediate frequency furnace and other commonly used straight in $10 \mathrm{kV}$ power distribution net, so the nonlinear load and as the direct input to the distribution of harmonic sources, will cause the current, voltage waveform distortion, distribution network harmonic generation. Harmonic produces serious pollution to power network, and even equipment in distribution network transformer, capacitor Banks, motor, voltage transformer, such as destructive, and lead to distribution network line loss increase[4, 5].

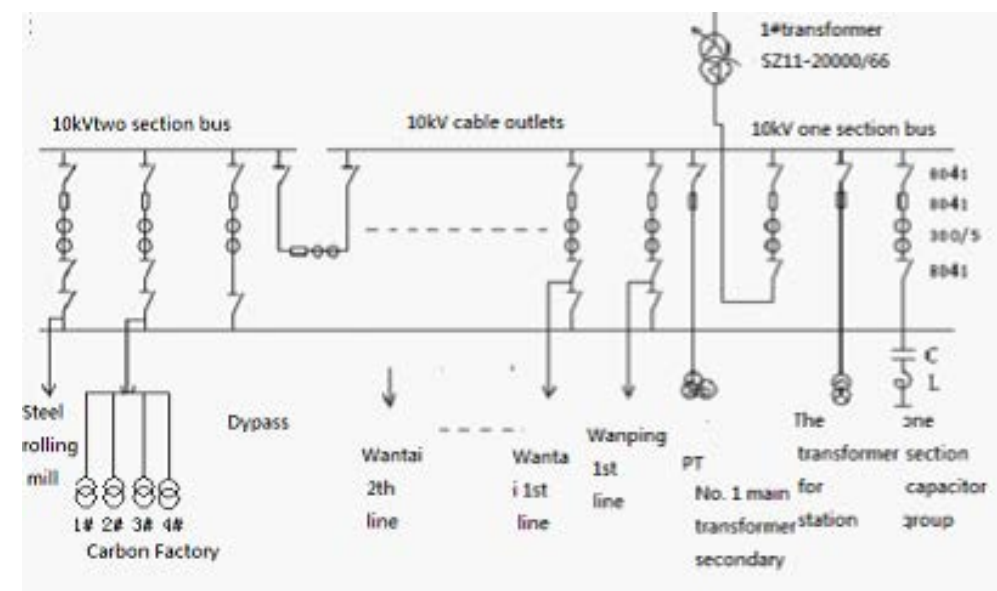

Figure 1 The $10 \mathrm{kV}$ substation system wiring diagram

\section{Harmonic harm to distribution transformer}

(1)Accident cases

Figure 1 is for a $10 \mathrm{kV}$ substation system wiring diagram, Oriental carbon factory main production machinery: crusher, milling machine, calcinations furnace, vibrating screen, press, 
kneading, kneading machine pan, etc. Oriental carbon plant a total of 4 units of 800 kva transformer (with 1 \#, 2 \#, 3 \# and 4 \#), on April 12, 2013, 2 about 50 points, 1 \# transformer gas protection action, 2 \# transformer oil smoke, carbon factory line switch trip. While creating square line with the user "xiangyang paper" a $630 \mathrm{kVA}$ appear a lot of different sound and oil temperature as high as $110 \mathrm{C}$ high temperature.

(2) Accident analysis

Most of Carbon Plant equipment is non-linear loads, resulting in a large number of harmonics in the production, not only pollute their own devices, while a harmonic source to $10 \mathrm{kV}$ bus inject harmonics, harmonic on the bus so they flow into the million square line, etc. other distribution outlet on. 2013, 23 April to 28 April, on the bus, carbon plant, line, etc. harmonics million square test, the following table 1 for harmonic test data $10 \mathrm{kV}$ bus.

Table 1 10kV substation busbar harmonic test data

\begin{tabular}{|c|c|c|c|c|c|c|}
\hline \multirow{2}{*}{\multicolumn{2}{|c|}{$\frac{\text { Name }}{\text { Time }}$}} & \multicolumn{3}{|c|}{\begin{tabular}{l|l} 
A transformer substation &
\end{tabular}} & The bue name & \multirow{2}{*}{$\frac{10 \mathrm{kV} \text { bus }}{\text { seconds }}$} \\
\hline & & \multicolumn{4}{|c|}{ April 24, 2013, 11 at 00 seconds to April 27, 2013, 09 at 00 seconds } & \\
\hline Harmonic frequency & $\operatorname{Min}(\mathrm{A})$ & $\operatorname{Max}(\mathrm{A})$ & The average(A) & 95\%probability value (A) & GB limit(A) & Qualify or not \\
\hline Total harmonic effective value & 2.725 & 9.004 & 5.086 & 7.861 & --- & --- \\
\hline Fundamental wave & 53.79 & 99.34 & 75.75 & 92.62 & $\begin{array}{c}--- \\
---\end{array}$ & $\begin{array}{c}--- \\
---\end{array}$ \\
\hline 2times & 0.024 & 1.533 & 0.192 & 0.326 & 3.200 & normal \\
\hline 3times & 0.023 & 1.514 & 0.102 & 0.196 & 2.600 & normal \\
\hline 4times & 0.040 & 1.544 & 0.098 & 0.150 & 1.620 & normal \\
\hline 5times & 1.650 & 6.870 & 3.581 & 6.132 & 2.600 & exceeded \\
\hline 6times & 0.041 & 1.309 & 0.099 & 0.142 & 1.080 & normal \\
\hline 7times & 1.579 & 5.449 & 3.166 & 4.453 & 1.860 & exceeded \\
\hline 8times & 0.023 & 1.012 & 0.078 & 0.108 & 0.820 & normal \\
\hline 9times & 0.036 & 0.910 & 0.215 & 0.329 & 0.860 & normal \\
\hline 10times & 0.019 & 0.778 & 0.050 & 0.071 & 0.660 & normal \\
\hline 11times & 0.383 & 2.310 & 1.300 & 1.912 & 1.180 & exceeded \\
\hline 12times & 0.019 & 0.559 & 0.052 & 0.073 & 0.540 & normal \\
\hline 13times & 0.042 & 1.479 & 0.592 & 1.032 & 1.000 & exceeded \\
\hline
\end{tabular}

Based on harmonic test results show that the current harmonic five, seven, 11 and 13 times more than the standards stipulated by the state, the excessive amounts of harmonic injected into power grid, the grid has been seriously polluted. When the harmonic effect on the transformer due to skin effect makes some parts of the transformer winding produce up to $700 \mathrm{C}$ high temperature. The oil chromatogram test below used to validate. Table 2 is an oil chromatographic test results "Xiangyang Paper" 630kVA transformer.

Table 2 Results "Xiangyang Paper" 630kVA transformer

\begin{tabular}{l|l|l|l|l|l|l|l|l}
\hline \multirow{2}{*}{ Date } & component & \multicolumn{2}{l}{ Content $(\mu \mathrm{l} / \mathrm{L})$} \\
\cline { 2 - 9 } & $\mathrm{H} 2$ & $\mathrm{CO}$ & $\mathrm{CO} 2$ & $\mathrm{CH} 4$ & $\mathrm{C} 2 \mathrm{H} 6$ & $\mathrm{C} 2 \mathrm{H} 4$ & $\mathrm{C} 2 \mathrm{H} 2$ & $\mathrm{C} 1+\mathrm{C} 2$ \\
\hline 2012.11 .8 & 152 & 139 & 670 & 1849.18 & 1138.97 & 3295.07 & 11.39 & 6294.61 \\
\hline 2013.4 .18 & 284 & 287 & 589 & 2199.56 & 1146.21 & 3332.05 & 10.26 & 6688.08 \\
\hline
\end{tabular}

Three-ratio method to determine:

Three ratio encoding: $\frac{C_{2} H_{2}}{C_{2} H_{4}}=0.003<0.1$, ratio is “ 0 ”; $\frac{C_{4}}{H_{2}}=7.7>3$, ratio is "2"; $\frac{\mathrm{C}_{2} \mathrm{H}_{4}}{\mathrm{C}_{2} \mathrm{H}_{6}}=2.9(1 \sim 3)$, ratio is “ 1 ”. 
Three ratios encoded as "0,2,1." This code is $300 \sim 700 \mathrm{C}$ temperature range overheating fault.

This subject to harmonic pollution Xiangyang paper 630kVA transformer in a large amount of harmonic, exceed the standard under the action of the transformer, forced outage.

\section{The harm of harmonic to capacitor group}

Harmonic harm to the reactive compensation capacitor is mainly manifested in the following aspects:

1) Capacitors due to the harmonic currents and overload, because the capacitance capacitor decreases with the increase of frequency, which makes the capacitor becomes the absorption point harmonic. At the same time, the harmonic current generated by the harmonic voltage will cause the capacitor fuse. 2) Harmonic tend to make the dielectric loss increase, which is a direct consequence of heat output of the capacitor group increased, shorten service life. 3) inductance capacitor may with the grid in combination, form a parallel resonance of a harmonic frequency, or make the harmonic were grossly enlarged, the final voltage will be significantly higher than the voltage rating, and result in damage of capacitors or fuse. 4) capacitor and may be combined with inductance in power network, harmonic frequency composition of a series resonant circuit, caused by harmonic current capacitor overload, resulting in damage of the capacitor.

(1)The accident case

In recent years, many accidents occurred frequently, the capacitor of the power supply company group such as imply village on July 5, 2011, a group of capacitor on July 30, 2011, on April 7, 2012, on April 10, 2012 successively trip. Accident phenomenon sometimes with capacitor outer blown fuse, the differential current of current transformer on $\mathrm{ZnO}$ arrestor explosion, etc.; On November 3, 2011 new substation, put in a hot standby $10 \mathrm{kV}$ reactive compensation capacitor group, after sending, series of reactor and capacitor Banks issued a terrible noise, $10 \mathrm{kV}$ bus bar a voice discharge at the same time, and was forced to shut down the condenser group [7]; On February 1, 2012, wu home fort A group of $10 \mathrm{kV}$ substation capacitors in explosion occurred in the operation of the trip, in A three phase capacitor bulge; B phase two capacitor burst collapse, two bulge; $\mathrm{C}$ is a deformation and cracking.

(2) Accident analysis

In 2012 November to Li Shizhai, Wan Xin, Wu Jia Bao substation harmonic test, Table 3, follows Li Shizhai results shown: (Wan Xin, Wu Jia Bao substation is almost the same with $\mathrm{Li}$ Shizhai).

From the test table can be seen, the big harmonic content (more than the national standard) is the cause of capacitor group accident.

Table3 Statistics of the harmonic content of Li Shizhai station

\begin{tabular}{c|c|c|c|c|c}
\hline Station & Line & Number & Max (A) & Nor (A) & Note \\
\hline \multirow{7}{*}{ Li Shizhai } & 3 & 2.686 & 5.300 & small \\
\cline { 2 - 6 } & Bus & 5 & 4.073 & 6.340 & closed \\
\cline { 2 - 6 } & Li Gang 1 & 3 & 2.457 & 4.900 & small \\
\cline { 2 - 6 } & Line & 5 & 7.454 & 5.900 & over \\
\cline { 2 - 6 } & Li Gang 2 & 3 & 2.708 & 5.370 & small \\
\cline { 2 - 6 } & Line & 5 & 6.135 & 6.420 & closed \\
\cline { 2 - 6 } & Li Tie 1 & 3 & 4.891 & 5.370 & closed \\
\cline { 2 - 6 } & Line & 5 & 9.409 & 6.420 & over \\
\hline
\end{tabular}

\section{Harmonics harm on motor}

(1) Accident cases

In June September, 2011, a power company constantly from enterprises and residents complaints, reflect the main problem with high and low voltage operation and mechanical CNC 
lathe operation state is not stable, the defective rate, and some production equipment can't normal start and work, and so on, the residents was mostly cannot normal startup and operation of home appliances, light, etc., including the dawn machinery factory has six motor is damaged. Through the complaint acceptance survey, found that these users are made by Yang Shigang $10 \mathrm{kV}$ bus power supply substation, the part of the system connection as shown in figure 2, one HuiDong, second line for power supply, HuiDong steel mills have 6 sets each line capacity of $1500 \mathrm{kw}$ intermediate frequency furnace.

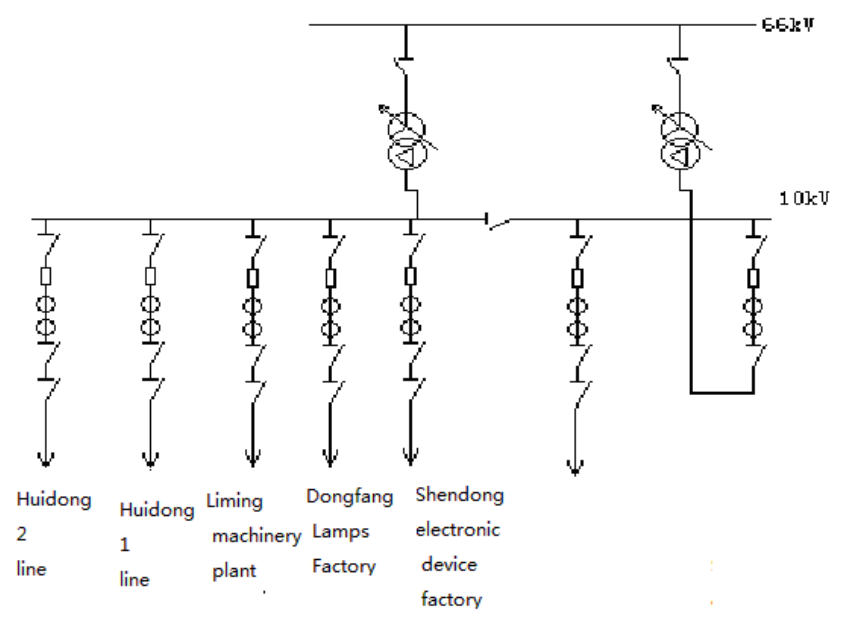

Figure 2 Yang Shigang substation wiring diagram (partial) system

(2)The reason analysis

The working principle of intermediate frequency furnace is the three-phase power frequency ac power after rectifying circuit is a dc power supply, through the inverter circuit output for single phase medium frequency $(1500 \mathrm{~Hz})$ ac power supply medium frequency induction furnace, and then to heat the metal. Intermediate frequency furnace at run time with high power factor, the characteristics of light load and low power factor, even the lowest power factor reaches 0.5 or so. Odd harmonics will produce in the operation of the time, when the 11, 13, 17 high harmonic current is large, will cause serious distortion, the power supply voltage is mainly characterized by high and low power factor, voltage fluctuation and flicker, etc.

Only 11 harmonics, for example a concrete analysis is as follows: 11 harmonics belongs to (time) of harmonic, phase sequence of negative sequence, negative sequence current transformer to power the entire system, for all power load in the system will produce adverse effects, especially the motor load. Are produced when the negative sequence current is flowing through the motor and the rotor of reverse phase, 2 times of power frequency magnetic field, make additional loss increase, resulting in additional temperature rise, at the same time can also cause mechanical vibration and noise of motor and harmonic overvoltage, and even lead to motor damage accident.

Figure 3 is on October 3, 2011 for Yang Shigang substation harmonic figure $10 \mathrm{kV}$ bus bar test.

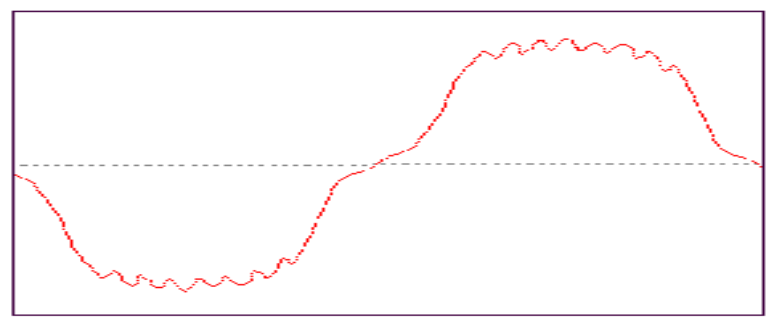

Figure 3 Yang Shigang 10 kv substation busbar harmonic measurement

\section{Harmonic harm to transformer}

Due to the distribution network using compensation mode, namely $\omega \mathrm{L}>1 / \omega \mathrm{C}$. When single-phase grounding distribution network occurs (external excitation conditions), voltage 
transformer fault current will flow through, if a user USES frequency equipment production points, because the frequency is low, omega $\mathrm{L}$ sharply reduced, is likely to reach omega $=1 \mathrm{~L} / \mathrm{C}$ conditions, omega frequency resonance voltage transformer is easy to occur. In addition, when the frequency is very low, omega L sharply decreases, and the exciting current increases rapidly, high frequency resonant voltage not only, also will appear on the inductor greatly over current (up to one hundred times the rated current), large current will produce a lot of electric power and temperature rise, its destructive power is very big, easy to burn inductance such equipment.

The accident case

10 o 'clock on September 20, 2012, 48 points, including $66 \mathrm{kV}$ youth two $10 \mathrm{kV}$ substation system in single phase grounding, 5110 points and $10 \mathrm{kV}$ distribution of pharmaceutical line tripping, at the same time, A voltage transformer high voltage side blown fuse, zero phase on the low voltage side blown fuse, transformer smoke, fuel injection, A bus three zinc oxide lightning arrester B phase thermal breakdown occurs, A, C two-phase overheating.

\section{Conclusion}

At present, the main distribution network harmonic source industry mainly has the following kinds: (1) electric arc furnace production industry. (2) of reversing cold rolling steel plate mill production industry such as iron and steel industry; (3) the electric railway use "make straight" type electric locomotive; (4) in heavy load industry such as the elevator; (5) large kneading machine industry.

\section{Reference}

[1] Shao Zongqi. Medium frequency furnace harmonic control. Electric technology: 2009, 11:58-63

[2] Zhang Yucheng, Du Mingzhe. Analysis and suppression. Central China electric power harmonic intermediate frequency electric furnace: 2006, 19 (1): 30-32

[3] Yang Hua, Wang Xiaoneng. Intermediate frequency furnace harmonic measurement and governance reform. Sichuan Electric Power Technology, 2005 1: 49-50

[4] Yang Bo, Cheng Richuang. Intermediate frequency induction electric furnace harmonic control and reactive power compensation design and application. Power Capacitor 2006, (5): 17-20

[5] Chen Huagang. Electrical equipment preventive test techniques for question answering. Hydraulic and electric power press, 1998 